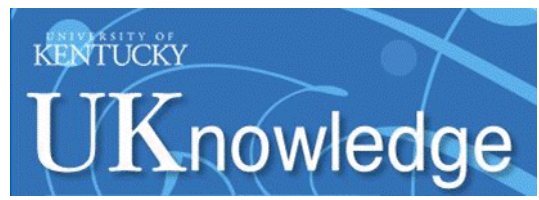

University of Kentucky

UKnowledge

Power and Energy Institute of Kentucky Faculty Publications

3-15-2021

\title{
Multi-Objective Optimization for Aircraft Power Systems Using a Network Graph Representation
}

\author{
Damien Lawhorn \\ University of Kentucky, damien.lawhorn@uky.edu \\ Vandana Rallabandi \\ GE Global Research, vandana.rallabandi@uky.edu
}

Dan M. Ionel

University of Kentucky, dan.ionel@uky.edu

Follow this and additional works at: https://uknowledge.uky.edu/peik_facpub

Part of the Power and Energy Commons

Right click to open a feedback form in a new tab to let us know how this document benefits you.

\section{Repository Citation}

Lawhorn, Damien; Rallabandi, Vandana; and Ionel, Dan M., "Multi-Objective Optimization for Aircraft Power Systems Using a Network Graph Representation" (2021). Power and Energy Institute of Kentucky Faculty Publications. 58.

https://uknowledge.uky.edu/peik_facpub/58

This Article is brought to you for free and open access by the Power and Energy Institute of Kentucky at UKnowledge. It has been accepted for inclusion in Power and Energy Institute of Kentucky Faculty Publications by an authorized administrator of UKnowledge. For more information, please contact UKnowledge@lsv.uky.edu. 


\title{
Multi-Objective Optimization for Aircraft Power Systems Using a Network Graph Representation
}

\author{
Digital Object Identifier (DOI) \\ https://doi.org/10.1109/TTE.2021.3066123 \\ Notes/Citation Information \\ Published in IEEE Transactions on Transportation Electrification.
}

(C) 2021 IEEE Copyright Notice. "Personal use of this material is permitted. Permission from IEEE must be obtained for all other uses, in any current or future media, including reprinting/republishing this material for advertising or promotional purposes, creating new collective works, for resale or redistribution to servers or lists, or reuse of any copyrighted component of this work in other works."

The document available for download is the authors' final manuscript version. The final published version is copyrighted by IEEE and is available as: D. Lawhorn, V. Rallabandi, and D. M. Ionel, "Multi-objective Optimization for Aircraft Power Systems using a Graph Network Representation," in IEEE Transactions on Transportation Electrification, doi: 10.1109/TTE.2021.3066123.

This article is available at UKnowledge: https://uknowledge.uky.edu/peik_facpub/58 


\title{
Multi-objective Optimization for Aircraft Power Systems using a Network Graph Representation
}

\author{
Damien Lawhorn ${ }^{1}$, Graduate Student Member, IEEE, Vandana Rallabandi ${ }^{2}$, Senior Member, IEEE, \\ and Dan M. Ionel ${ }^{1}$, Fellow, IEEE \\ ${ }^{1}$ SPARK Lab, Department of Electrical and Computer Engineering, University of Kentucky, Lexington, KY, USA \\ damien.lawhorn@uky.edu, dan.ionel@uky.edu \\ ${ }^{2}$ GE Global Research, Niskayuna, NY, USA, vandana.rallabandi@ieee.org
}

\begin{abstract}
Today, the electrification of flight is more popular than ever, creating a wide array of concept aircraft and associated power system topologies. In order to gain insights into benefits of these varying architectures, this paper introduces the development of a framework for electric aircraft power system (EAPS) optimization. The proposed framework accepts inputs from a designer in the form of component parameters and desired flight mission characteristics. A collective graph representing many possible architectures is formed, from which, subgraphs that describe power system topologies meeting the flight requirements are extracted and analyzed. Optimal EAPS architectures with respect to goals of minimizing mass while maximizing efficiency and reliability can be subsequently selected from these subgraphs. The framework is exemplified on a $500 \mathrm{~kW}$ rated aircraft using data collected from surveys of component parameters such as power density and efficiency. The presented results show a comparative analysis of different EAPS types with respect to the competing performance metrics of mass, efficiency, and survivability.
\end{abstract}

Index Terms - graph theory, electric aircraft, power systems, optimization

\section{INTRODUCTION}

Electric aircraft are becoming increasingly more popular, driven by incentives such as fuel efficiency, cost, and noise pollution. Manufacturers have already begun electrification of many auxiliary systems in today's planes, as well as the demonstration of electrically propelled aircraft. These studies have produced a large number of designs, all of which vary greatly with respect to the power system (Fig. 1). The question of how these different architectures may perform has led to the development of a tool which can be used to determine the optimal number of components and configuration within the power system.

When considering the electrification of air transport, the EAPS architectures may be broken down into three main categories: all-electric, hybrid-electric, and turbo-electric. The first of these mentioned include a propulsion system which fully derives its energy from electrical storage means. With the current state of the technology's maturity, all-electric aircraft concepts are most commonly seen. In fact, several demonstrator aircraft have been constructed or are currently under development.

One of the first all-electric demonstrator aircraft to attract significant attention was the E-Fan developed by Airbus [1]. This $60 \mathrm{~kW}$, two-seater prototype was the only one produced and first flew in 2014, before Airbus transitioned to the Authors' final manuscript version. The final published version is copyrighted by IEEE and is available as: D. Lawhorn, V. Rallabandi, and D. M. Ionel, "Multi-objective Optimization for Aircraft Power Systems using a Graph Network Representation," in IEEE Transactions on Transportation Electrification, doi: 10.1109/TTE.2021.3066123. (O2021 IEEE Copyright Notice. "Personal use of this material is permitted. Permission from IEEE must be obtained for all other uses, in any current or future media, including reprinting/republishing this material for advertising or promotional purposes, creating new collective works, for resale or redistribution to servers or lists, or reuse of any copyrighted component of this work in other works." development of their hybrid-electric aircraft concept. More recently, electric propulsion company, MagniX partnered with AeroTec to modify a Cessna 208B Grand Caravan to be electrically driven by MagniX's 560kW system in 2020 [2]. Another notable unique all-electric currently under development is NASA's all-electric X-57 (Fig. 1). This aircraft will not only serve as a demonstrator for electric machines in aviation, but also for a concept called distributed electric propulsion (DEP), which, according to studies, may increase flight efficiency further. While many organizations are showing that purely electric flight is a possibility, all-electric aircraft are typically seen to be short range and carry a limited number of passengers, due to the current limitations with lithium-ion energy storage energy density.

As current electrical energy storage means limit maximum payload and flight time in all-electric aircraft, researchers have proposed alternative approaches to incorporate further electrification into aircraft propulsion systems. The most commonly seen method of driving propulsion in modern commercial aircraft is through combustion jet engines, which is a proven technology that the industry has become well suited to manufacture. Electrification of these propulsion systems type may be achieved through the coupling an electrical generator to the turbine of a jet engine. This method allows the jet engine to perform its duty of producing lift while simultaneously generating electrical energy. A hybrid-electric aircraft power system includes significant energy storage means for the generated electricity, which may be used to drive electric machines connected to propulsors at any period during the flight. One hybrid-electric aircraft example concept is the EFan X, a collaborative effort between AirBus, Rolls-Royce, and Siemens [3].

Turbo-electric aircraft, in comparison, directly use the power produced by the jet turbine to drive the electrical propulsion motors, including little to no means of electrical energy storage. Turbo-electric concepts generally offer a less massive design when compared to a hybrid considering the very small amount of battery used. This, however, makes the power system less flexible and decreases its potential for reduction of direct carbon emissions. Example turbo-electric aircraft concepts include NASA's Single-aisle Turbo-electric Aircraft with an Aft Boundary-Layer propulsor (STARC-ABL) and N3-X [4], [5].

At the preliminary design stage, an aircraft's mission is typ- 
ically predetermined based on flight goals such as endurance, intended payload, etc. Generally, the aircraft topology of turboelectric, hybrid-electric, or all-electric may be set based on its mission duration, primarily due to energy storage constraints. The leading electrical energy storage technology in electric vehicles, Lithium-ion, may possess a specific energy in the range of about $160 \mathrm{Wh} / \mathrm{kg}$ [6].

Fossil fuel, with an energy density two orders of magnitude higher, holds an advantage over electric energy storage. Thus, when considering electrical energy storage for a commercial airliner such as the Boeing 777, it may be expected that significantly more mass is required to achieve the same energy content as fossil fuel, and this severely limits the maximum flight time.

There are still many choices for a designer to make concerning the layout of a power system, including the degree of electrification, distribution type, number and rating of power conversion devices, energy storage systems, and propulsion units such as motors and jet engines [7]. Determination of these free variables in the process is not a trivial question and requires detailed analysis.

In past EAPS design studies, a common approach is to construct a small set of candidate architectures and evaluate performance metrics compared to a baseline, such as the approach seen in [8]. When considering propulsion design, aerodynamics, thermal, and electrical systems are highly coupled. Multidisciplinary tools such as GT-HEAT of Georgia Institute of Technology, and GENUS of Cranfield University have been developed to assess integrated vehicle impacts of various subsystems [9], [10]. While, graph theory based methods have been employed recently for the design of microgrids and large electric ships to evaluate system reliability and cost, the applications to EAPS is less widely researched [11], [12].

This paper proposes an approach, intended to be used as an early stage design tool, which utilizes mathematical methods involving graph theory to identify optimal solutions with regard to the electric aircraft power system (EAPS) configuration built off of the initial work conducted in [13]. Our paper brings contributions, not only in applications to electric aircraft, but also in the theoretical development and the software implementation, as explained later in the paper. Example results include performance trade-offs for an array of design candidates in the terms of efficiency, mass, and survivability.

The paper is organized as follows. Section II presents considerations and a review of components commonly seen in EAPSs. Section III discusses the proposed optimization method based on predefined mission parameters including components characteristics outlined in the previous section. An example case study for a $500 \mathrm{~kW}$ airplane using the framework is presented in Section IV. Based on the results from the case study, architecture performance sensitivity to the various components is discussed in Section V. The full paper is concluded in Section VI.

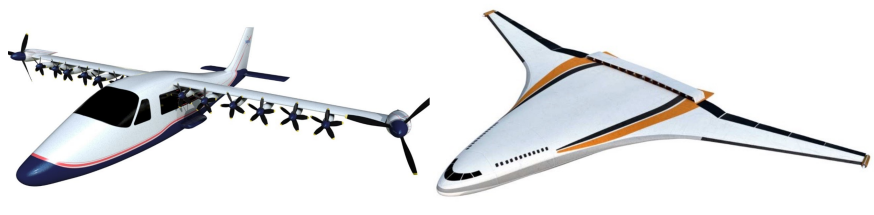

Figure 1. Example distributed electric propulsion aircraft designs by NASA with varying power system topologies. All-electric demonstrator X-57 Maxwell currently under development utilizing multiple electrical machines with varying ratings [14] (left). Conceptual turbo-electric N3-X aircraft which utilizes electrical energy from generators coupled to jet engines [15] (right).

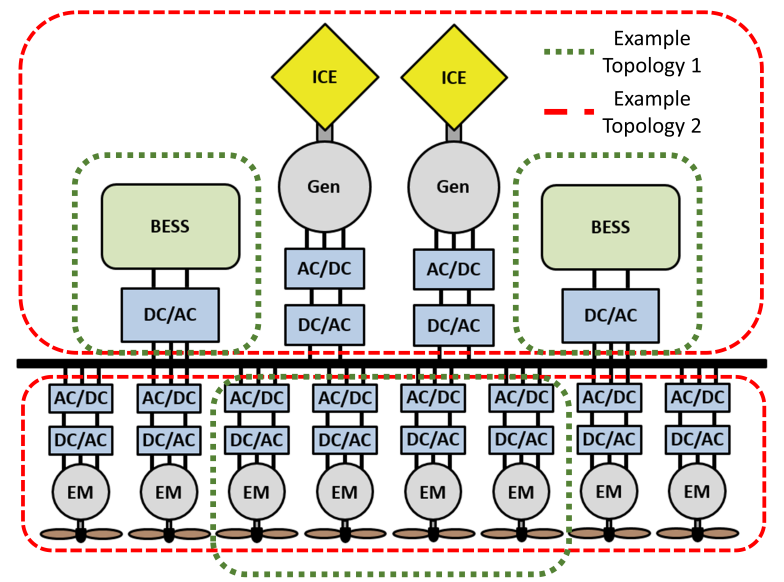

Figure 2. Example aircraft power system representations with a variable number and ratings of internal combustion engines (ICE), generators (Gen), rectifiers (AC/DC), inverters (DC/AC), battery energy storage systems (BESS), and electric motors (EM). The proposed graph theory based optimization approach identifies high performance candidates from the most general of architectures.

\section{Electric Aircraft Propulsion Component SURVEY}

An EAPS may be represented in the form of a graph network in which the graphs nodes are determined by the components incorporated in the power system architecture. For an electric aircraft, these may include, but are not limited to electric machines, i.e. motors or generators, power converters, energy storage devices, and protection equipment (Fig. 2). Each component and its respective graphical node may be assigned characteristics including specific power or energy, operating efficiency, and failure rates. These characteristics may be constant values, such as a failure rate for a particular component, or they may vary with other variables related to that component. For example, efficiency may be calculated as a function of the component's power rating and anticipated loading over the specified mission. In this study, surveys have been conducted to extract these parameters from both commercially available components and those reported in the literature.

\section{A. Internal Combustion Engines}

Airplanes are traditionally driven with one of two powerplants types to generate thrust. Reciprocating, or piston engines can commonly be seen on fixed-wing propeller aircraft 

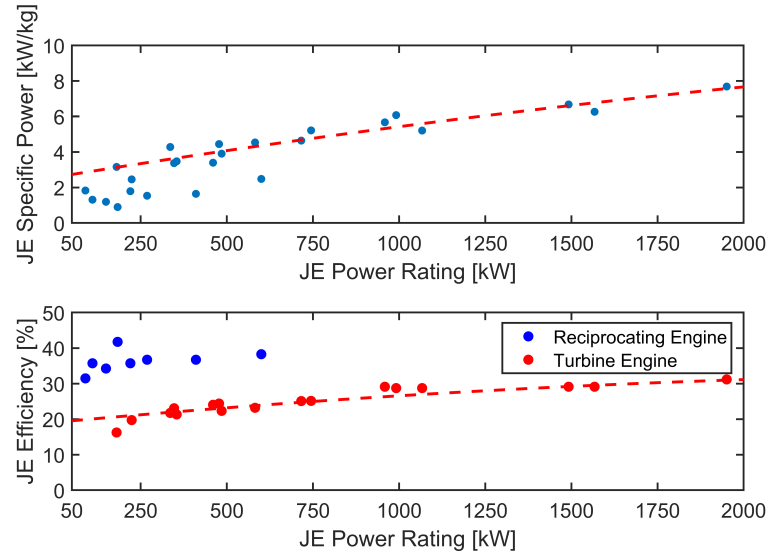

Figure 3. Survey of specific power and efficiency values for commercial jet engines, including both turbine and internal combustion engines based on a large set of references [16]-[22]. Trendlines shown are used in the EAPS model.
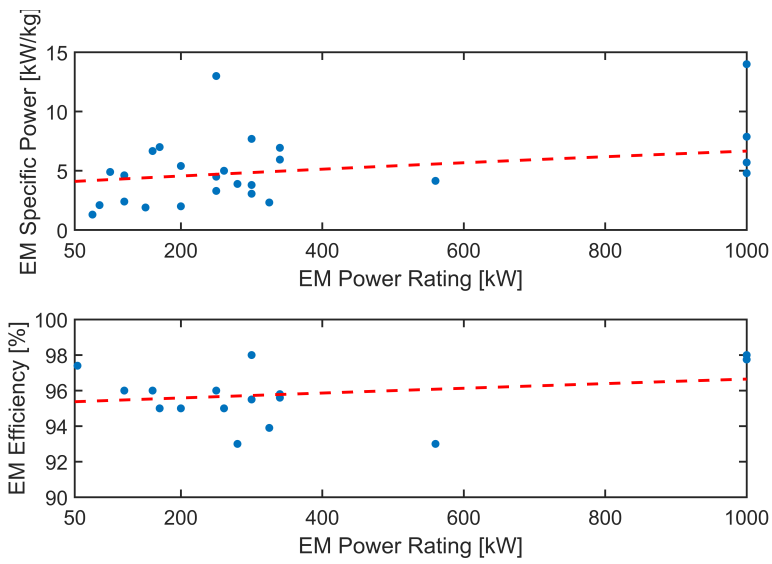

Figure 4. Collection of specific power and efficiency values for both academic and commercial electric machines designs based on a large set of references [23]-[36]. A trendline is extracted and used in the EAPS model.

or helicopters and are generally more inexpensive and easier to operate. These engines are used in lower power aviation applications such as training and short-haul missions. For use in higher power demand applications, aircraft adopt a power turbine. In fixed-wing designs a turboprop or jet turbine may be used, while in helicopters utilize turboshaft engines. An extensive survey was conducted to gather parametric data across commercial aviation reciprocating and turbine engines [16]-[22]. From this survey, second order polynomial fitting was used to represent the variations in specific power and efficiency with rated power, as illustrated in Fig. 3. Turbine internal combustion engine (ICE) efficiency in percent, $\eta_{I C E}$, was estimated using the following equation;

$$
\eta_{I C E}=-1.46 * 10^{-6} P_{I C E}{ }^{2}+8.95 * 10^{-3} P_{I C E}+19.08,
$$

where $P_{I C E}$ represents the rated power of the ICE in $\mathrm{kW}$. Similarly, the power-to-weight ratio of the engine can be represented as

$$
s p_{I C E}=-3.03 * 10^{-7} P_{I C E}{ }^{2}+3.15 * 10^{-3} P_{I C E}+2.57,
$$

where $s p_{I C E}$ is the specific power in $\mathrm{kW} / \mathrm{kg}$.

\section{B. Electric Machines}

Electric machines may serve several functions in an electric aircraft system. The most commonly envisioned propulsion application is the direct linkage of the electric machine to the propulsive device, be it a propeller or ducted fan. In this application the machine mainly operates in motoring mode, working to drive the propulsive device to generate lift for the aircraft. However, regeneration and emergency braking is also achievable by the device. In hybrid-electric and turbo-electric aircraft, electric machines may be coupled to the turbine of jet engines to serve as generators, providing electrical power to the energy storage or directly to motoring electric machines. In this configuration, the electric machines may also be used as starters for the jet engines. For use in the optimization framework, a collection of performance parameters in electric machines designed for vehicle applications was constructed, using the assumption only active mass has been reported (Fig. 4). The majority of electric machines used in transportation applications and for which data is publicly available, fall in power ranges below $500 \mathrm{~kW}$ [23]-[36]. The specific powers and rated efficiencies can vary significantly, as illustrated in Fig. 4. Therefore, if a least squares regression line was utilized as described for the combustion engines, a poor correlation may be the result. The efficiency and specific power are considered to increase with power rating, The goodness factor defined as the equation below:

$$
G=\frac{2 p^{2} \mu_{o} f}{\pi p_{r} g},
$$

serves a dimensionless index which can be used to compare electric machines of different ratings [37]. Where $p$ is the polepitch, $p_{r}$ is the surface resistivity, and $g$ is the air-gap length. It was shown that higher rated machines have a higher goodness. Further, as the power rating increases, inactive materials make up a smaller percentage of the overall weight, leading to higher specific power. Likewise, auxiliary losses comprise a smaller percentage of the overall loss. In addition, the probability of using expensive, low loss components is higher, all these factors contribute to a higher efficiency. Based off of the survey data, it has been assumed in this study that the powerto-weight ratio of electric machines increases linearly from 5 to $10 \mathrm{~kW} / \mathrm{kg}$ as the power rating increases to $2.5 \mathrm{MW}$. Similarly, the efficiency over this power range was assumed to increase linearly from 95 to 98 percent. Power rating of the electric machines has been determined based on the power requirements dictated by the mission profile, for the case study presented in this paper, the aircraft has a total power rating of $500 \mathrm{~kW}$. Second order effects of fan drag, efficiency, and weight are neglected.

\section{Power Electronics}

Each electric machine represented in the aircraft designs must be utilized with the implementation of power electronic 


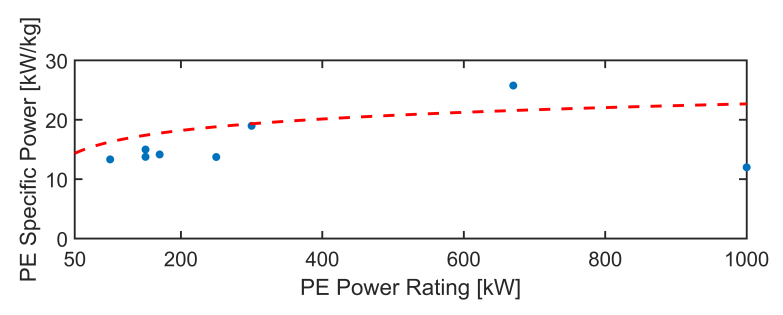

Figure 5. Survey of specific power and efficiency values for commercial and academic power electronic conversion devices, based on a large set of references [38]-[44]. Trendlines shown are used in the EAPS model.

converters to condition the power where appropriate. A survey of performance characteristics for various industrial and academic power electronic converters has been conducted and is illustrated in Fig. 5. The survey consists solely of designs for three-phase, two-level inverters due to the fact that such a topology is the most common implementation for an electric vehicle motor drive application. Additional detail could be added to the modeling by including survey results for various motor and drive combinations. A logarithmic fitting has been used to generate a least squares regression line from the data points and is used in the optimization. Specific power, $s p_{P E}$, of the power electronic converters, or the power-to-weight ratio, has been estimated as

$$
s p_{P E}=3.47+2.78 \log \left(P_{P E}\right),
$$

where $P_{P E}$ is the rated power of the power electronics. Typically, in matured technologies and applications of electric machines and power electronics, efficiency is expected to increase with rated power. These trends are noted in common practice and have theoretically demonstrated in the following paper [37]. With EAPS technologies, there is large variation in between technological designs, which may explain the variations in the survey data. Due to the lack of efficiency reported by manufacturers, in this study the efficiency of the power electronics is assumed to follow a linear increase from 97 to 99 percent as power increases to $1 \mathrm{MW}$.

\section{Energy Storage}

The studies conducted in this paper and previous works show that energy storage systems are among the most influential components included in the design of an electric aircraft power system when considering mass. Although primarily dominated by Lithium-Ion battery technology, there are many studies on implementation of energy storage in electric transportation including supercapacitors, various forms of chemical batteries, fuel cells, and flywheels. In this study it is also important to consider the energy stored in jet fuel used in a hybrid topology. A survey of recent review papers on energy storage for electric vehicle applications was conducted and performance characteristics are illustrated in Fig. 6. As shown in Fig. 6, power and energy-to-weight ratios vary between energy storage technologies. Therefore, when calculating the mass of electrical energy storage, both power and energy ratings are considered. In most applications, the energy rating is the dominate factor. However, in fuel cells for example,

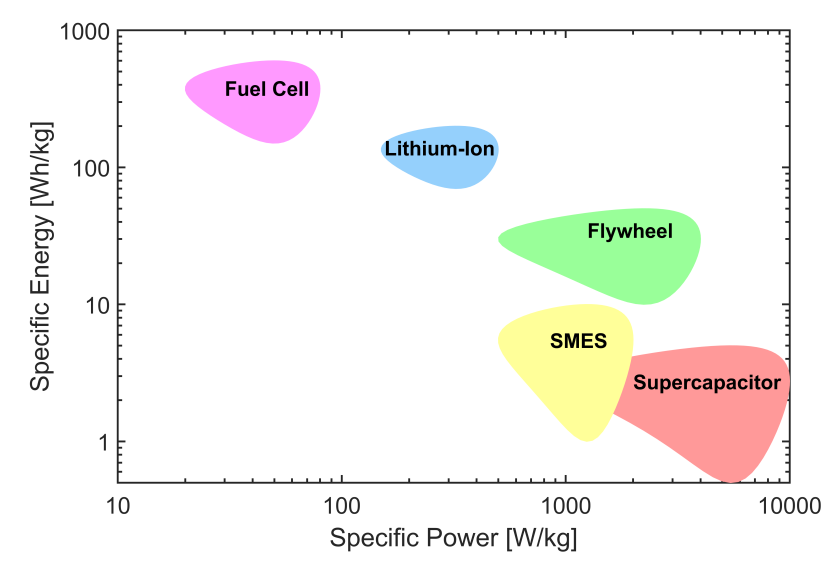

Figure 6. Ragone plot illustrating specific energy and power of various energy storage means usable in electrical transportation based off of references [45]-[47]. Survey conducted includes hydrogen fuel cells, lithium ion batteries, flywheels, superconducting magnetic energy storage (SMES), and supercapacitors. While a higher specific energy is typically favorable in electric propulsion, the specific power of the energy storage means must also be considered.

power rating may dictate mass, considering specific energy is higher than the specific power when compared to other technologies. Architectures which can make use of high power, lower energy applications such as energy recovery during landing, may favor storage means which have show higher specific power. For Lithium-ion batteries, which are based on a technology under accelerated development, relatively large variations of the specific energy with rating have been reported, making it difficult to conclude on an analytical relationship for the trends. For the studies reported in this paper, it has been assumed that the specific energy for the battery remains constant at the typical value of $160 \mathrm{Wh} / \mathrm{kg}$ [6].

\section{E. Circuit Protection}

Circuit breakers can be mechanical or solid state devices. Mechanical breakers (vacuum, SF6, air chute) are conventionally designed for $\mathrm{AC}$ applications. In order to use them in DC systems, an artificial current zero-crossing is achieved through passive and active resonant circuits. Such breakers feature a low contact resistance, and can be designed to withstand high voltage, but have large fault clearing times, of the order of milliseconds, and a large number of moving components. In contrast solid state breakers, based on thyristors, IGBTs, etc. have operation times of the order of micro and nano seconds [48]. One limitation is the high on-state loss, which can be mitigated by the use of wide band gap devices. The available current and voltage ratings are limited, necessitating, for high power applications, several devices to be connected in series, parallel or both, and their synchronous operation. This additional complexity may favor power system topologies with a large number of lower rated components.

\section{Exhaustive Design Space Evaluation Process}

The edges of a graph represent interconnections between the various components. A graph for an EAPS is directed to signify possible directions of power flow. Some nodes such 


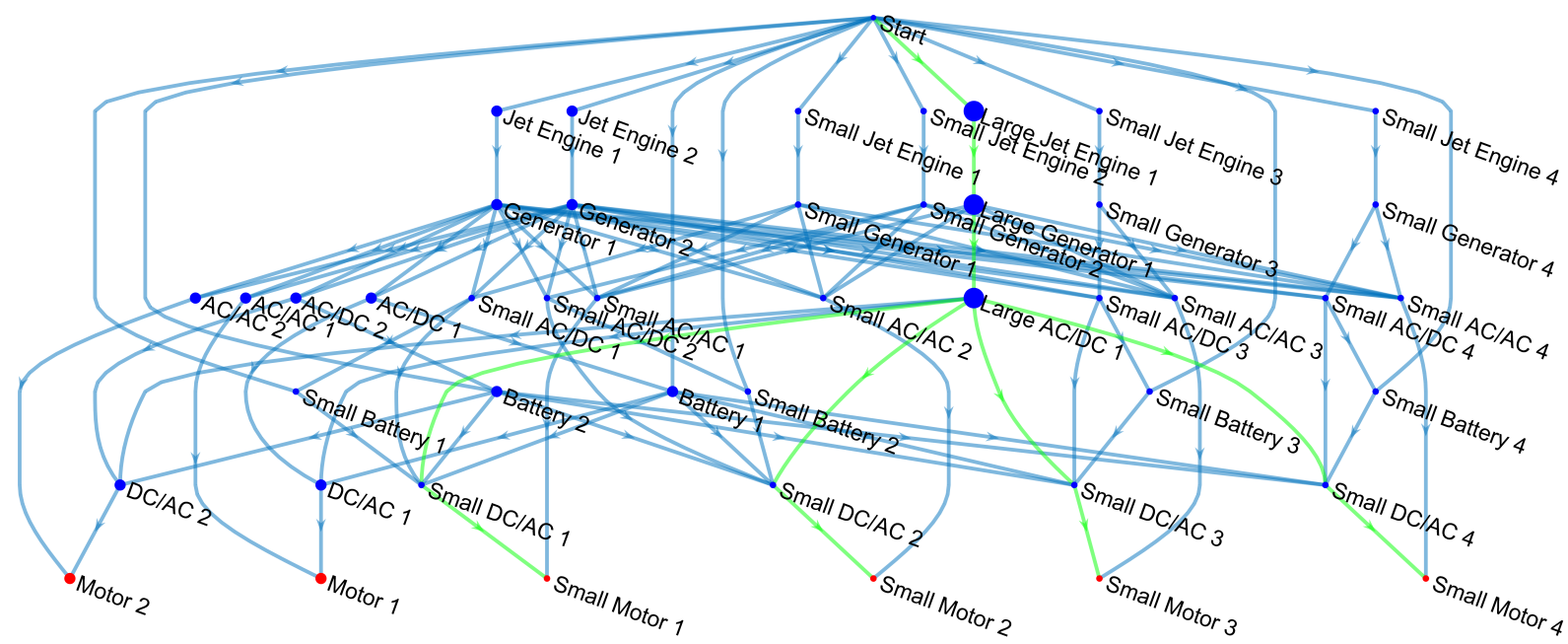

Figure 7. Graph containing power system components seen in hybrid and turbo-electric aircraft topologies with varying ratings. The illustrated graph represents thousands of potential candidate designs. The developed framework extracts valid subgraphs meeting the flight requirements, such as the one shown in green, for further analysis.

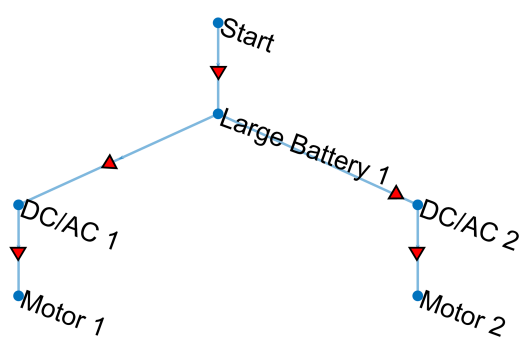

D1: All-electric

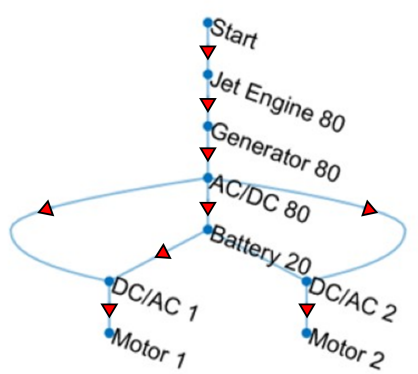

D4: Parallel Hybrid-electric

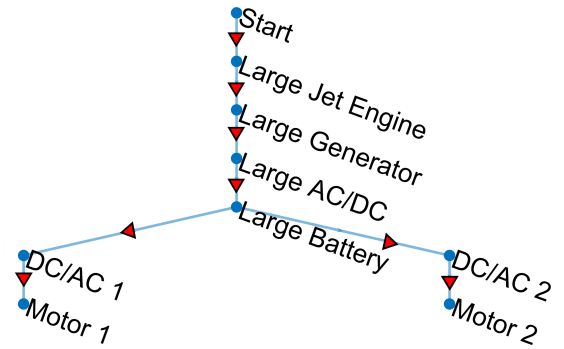

D2: Series Hybrid-electric

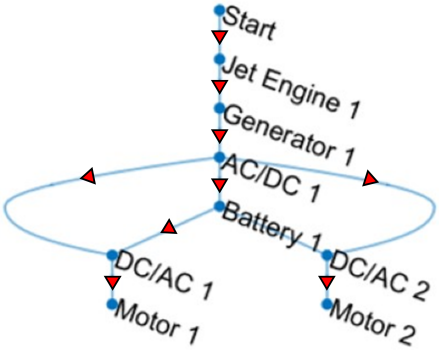

D3: Parallel Hybrid-electric by the system designer. All the shown topologies were selected automatically as subgraphs from a graph containing many designs. These architectures include purely turbo-electric, hybrid-electric and all-electric energy storage means.

as an auxiliary heating unit may only accept power, therefore its edges should only be directed toward and not from the node. These edges may be assigned weights, examples include mass associated with the inclusion of a particular vertex, power flow capacity, or physical distance. Further descriptions of how power systems may be represented as graphs can be seen in [49], [12]. Connectivity in between the various graph vertices may be used for minimal path calculation as in this study to determine lowest mass or highest efficiency. Additional studies in the literature have used graph theory for reliability evaluation by utilizing minimum cut sets to determine power loss scenarios [50].

The optimization process begins with inputs from the system designer regarding the intended use of the aircraft and technology available. Vehicle goals such as desired fuel savings, power requirement at maximum lift, and flight profiles, which describe aircraft power output over time, may be initially introduced by the user and later used as constraints. In addition to these vehicle-level inputs, the user also must define component-level information for the framework to use. These attributes include specific power and energy, efficiency, and failure rates. These may be defined static values as 
described earlier, or by ranges depending on the availability of resources to the aircraft designer. Introduction of ranges for component attributes also enables the use of sensitivity analysis to determine which components and attributes have the largest influence on performance metrics. In this study, a survey of various aircraft components was conducted to obtain values for efficiency and specific power as a function of power (Fig. 3 and Fig. 4). To further the accuracy of this model, additional power system components such as gearboxes can be modeled, as seen in [51].

Once the components under consideration and the desired ranges are defined, a graph is constructed to represent many candidate designs, as seen in Fig. 7. An iterative process is established for the addition of commonly seen power flow paths into the graph to avoid excessive manual entries by the user. One example of a common path starts from a jet engine as a generation source, which converts mechanical energy to electrical through an $\mathrm{AC}$ generator, from this point the power can be rectified with a AC/DC converter, then this power can be used to drive an AC. As an example, the conceptual design for a $500 \mathrm{~kW}$ aircraft will now be demonstrated. The system graph is iteratively created using components with varying power ratings, those labeled "large" represent a full power rating of $500 \mathrm{~kW}$, those with no indicator possess power of half the rated value, and those with "small" indicate a quarter of the power rating.

From the full graph, thousands of architectures are extracted which meet the power and energy requirements input by the user, such as those seen in Fig 8. To perform this extraction process, node are classified into power delivery (PD), energy source (ES), and power conversion groups. Jet engines are treated as both ES and PD nodes since fuel storage is included in the node characteristics. All possible permutations of ES and PD nodes are then calculated and stored into matrices, as illustrated in Fig. 9. The framework then iterates through the matrices to remove any permutations whose total power and energy capacity do not meet the requirements imposed by the designer, which may be described as the following equation:

$$
\begin{gathered}
P_{r e q}=\sum_{i=1}^{n_{E M}} P_{E M, i}+\sum_{i=1}^{n_{J E}}\left(P_{J E, i}-P_{L, i}\right), \\
E_{r e q}=\sum_{i=1}^{n_{B A T}} E_{B A T, i}+\sum_{i=1}^{n_{J E}} E_{J E, i},
\end{gathered}
$$

where $P_{r e q}$ is the maximum power and energy required for the aircraft's mission, $P_{E M}, E_{E M}, P_{J E}, E_{J E}$ and $E_{B A T}$ are the rated power and energy of the electric motors, jet engines, and batteries, $P_{g}$ is the generated electrical power from a jet engine's turbines and $n_{E M}, n_{J E}, n_{B A T}$ are the number of components in the design candidate. Subgraphs are then formed by using the previously defined ES and PD nodes in combination with minimum path algorithms to add edges and power conversion nodes. Additionally, the algorithm ensures the following power flow constraints between one a parent and daughter node(s):

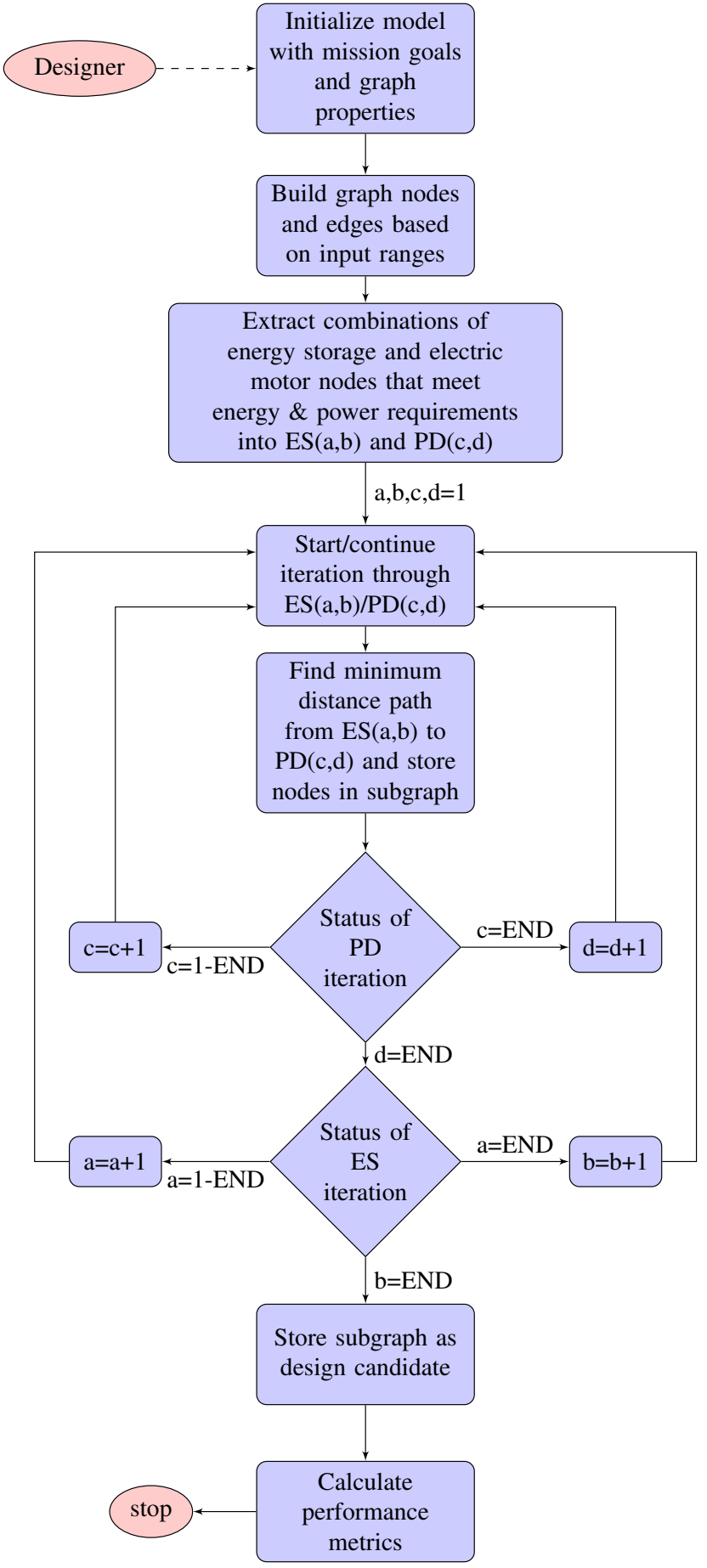

Figure 9. Workflow for the EAPS optimization process. The designer begins the approach by defining system characteristics and the framework outputs how viable power systems perform based on specified metrics. Example performance metrics include mass and efficiency.

$$
P_{s}=-\sum_{i=1}^{n_{l}} P_{l} ; \quad P_{s}<0 ; \quad P_{l}>0,
$$

using the convention of supplied power being noted as negative and absorbed power as positive, where $P_{s}$ is a parent node and $P_{l}$ is a daughter node.

These architectures that are extracted include the extreme 


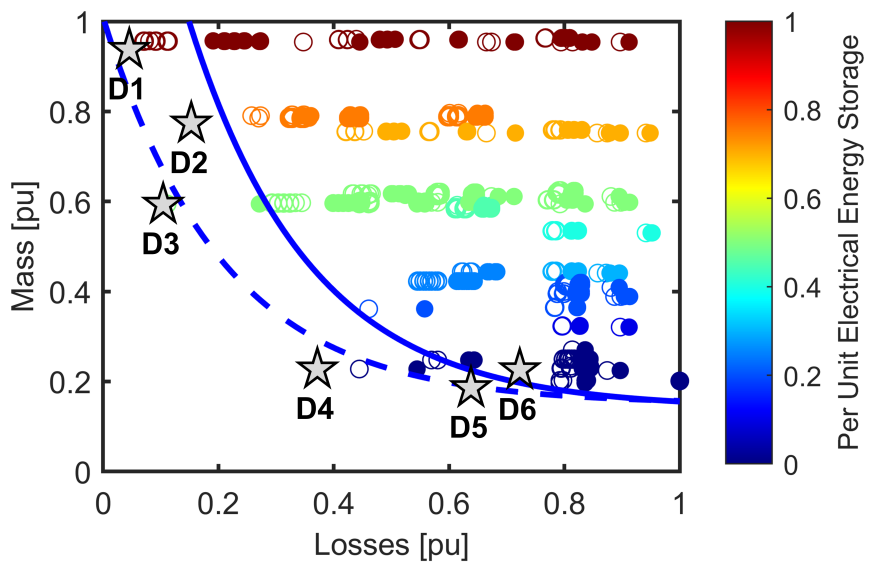

Figure 10. Total mass and losses for the entire power system of aircraft including combustion engine, electric generation, energy storage, power conversion and electric propulsion motor are shown as empty circles. Filled circles indicate designs that consider both power system and propulsion losses. The dashed and solid lines indicate generic pareto fronts for both calculations. Designs with purely electrical energy storage have the highest efficiency (D1) and turbo-electric designs are least massive (D5 and D6). Hybrid designs such as D2-D4 tradeoff mass and losses.

cases of all-electric and turbo-electric, which offer the most efficient and lightest designs respectively, and also series hybrid and parallel hybrid-electric aircraft power systems, which offer best case compromises. Design D1 is an purely electric topology with a battery rated for $500 \mathrm{~kW}$ and two $250 \mathrm{~kW}$ motors and associate drives. Design D2 is a series hybrid with a $500 \mathrm{~kW}$ jet turbine powering a similarly rated generator, rectifier, and battery, which then supplies two $250 \mathrm{~kW}$ motors and drives. Designs D3 and D4 are parallel hybrids with a $250 \mathrm{~kW}$ jet engine supplying a generator, rectifier, and battery of the same rating. D3 and D4 differ in the amount of energy stored in the battery and fuel tank, where D3 uses 50 percent of each and D4 uses 80 percent stored in the engine and 20 percent from the battery. Designs D5 and D6 are turboelectric systems with no electrical energy storage, both utilizing a $500 \mathrm{~kW}$ engine and generator. D5 is a more distributed approach, which may prove more reliable and exhibit increased aerodynamic efficiency with four $125 \mathrm{~kW}$ rectifier, inverter, motor combinations. D6, in contrast, has a single $500 \mathrm{~kW}$ rectifier with two $250 \mathrm{~kW}$ inverters and motors.

\section{Discussion of Example Studies}

A collection of valid subgraphs was constructed using the optimization approach discussed. Once obtained, postprocessing may be done to evaluate the performance characteristics of each subgraph representing the aircraft power system topologies. In this study, two example studies were conducted to assess architecture performance on the basis of mass, efficiency and survivability. To further validate the design candidates produced by the framework, multi-physics co-simulation of the architecture may be conducted, as seen in [52].

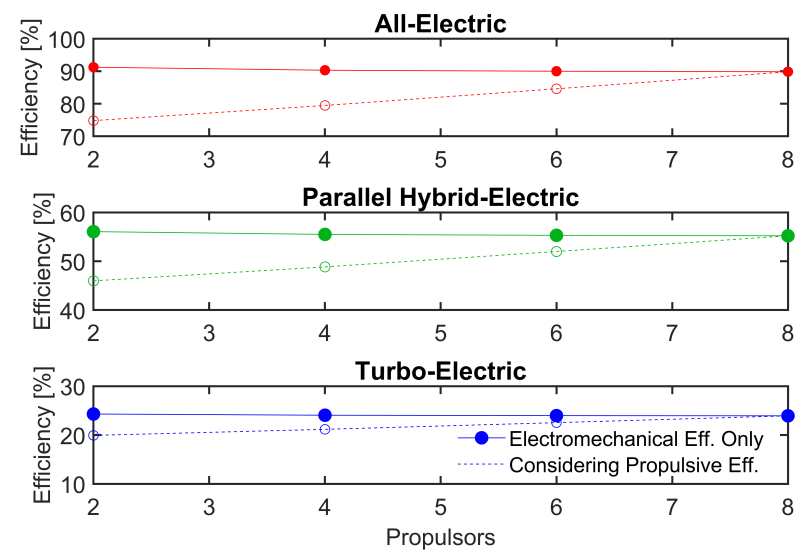

Figure 11. Distributed electric propulsion (DEP) effects on total system efficiency. When only the electromechanical efficiency is considered, losses increase with the number of propulsors due to the decrease in component efficiency as the power rating decreases. If the benefits of DEP are also considered, the overall system efficiency increases with the number of propulsors.

\section{A. Evaluation of Mass and Efficiency}

The total power system mass may be calculated as the sum of all individual components masses and any energy storage. Nodes that represent loads or power conversion devices derive their mass from the node's rating and the associated specific power. The mass for those nodes which represent Lithium-ion batteries, is calculated using specific energy. A special case is introduced for jet engine nodes, which represent both the engine and fuel storage. Total ICE node mass is calculated as the sum of engine mass, based on specific power, and mass of the required fuel. The mass of fuel used by an ICE per energy output is known as specific fuel consumption (SFC). Based on the survey conducted in Section II A, a second order polynomial fitting has been used to estimate a turbine's SFC as a function of power rating:

$$
C_{I C E}=2.14 * 10^{-8} P_{I C E}-1.27 * 10^{-4} P_{I C E}+0.42 \text {, }
$$

where $C_{I C E}$ is $\mathrm{SFC}$ in $\mathrm{kg} / \mathrm{kWh}$. To determine the required energy, a mission profile was used, which defines power consumption of the aircraft over time.

Another very important performance metric that is of interest to an aircraft designer is the overall system efficiency, which directly effects fuel consumption. Furthermore, the power losses determine the sizing for the thermal management and cooling. Recent studies have shown that distributed propulsion with multiple propellers and their associated electric machines may provide a higher aerodynamic efficiency [53]. Based on this reference, in a 250 PAX twin-jet, changing the number of engines from two to eight may increase the propulsive efficiency from 80 to 86 percent [54]. Although aerodynamic efficiency is expected to increase by means of boundary layer ingestion, as discussed in a number of references [53], [54], there isn't a clear analytical relationship established yet. In our current study, for the purpose of simplified exemplification, it has been assumed that an increase 
in efficiency from 80 to 86 percent occurs linearly as number of propulsors increases from two to eight.

$$
\eta_{\text {sys }}=\frac{\left.E\left(\eta_{\text {comb }}(1-\zeta)\right)+\left(\eta_{\text {elec }} \zeta\right)\right) \eta_{\text {aero }}}{E},
$$

where $\eta_{c o m b}$ represents the efficiency of a path from the combustion engine source to a motor load, $\eta_{\text {elec }}$ represents the efficiency of a path stemming from battery energy storage, $\zeta$ is the degree of electrical energy storage, being a value from zero to one and $\eta_{\text {aero }}$ is the aerodynamic efficiency. For a fully turbo-electric architecture, $\zeta$ will be equal to zero, as all the energy used for flight is stored in the jet fuel. It is assumed that the battery is charged independently of the engine.

This framework example study is conducted for a $500 \mathrm{~kW}$ peak power electric aircraft. The total system efficiency versus EAPS mass was calculated using multiple values of $\zeta$ and both, with and without, the propulsive efficiency considered. The results, shown in Fig. 10, indicate that higher efficiency is achieved at the cost of mass in systems with battery energy storage, which is in line with expectations, and therefore confirms the applicability of the proposed approach.

In the case where assumed benefits of distributed electric propulsion (DEP) are included in the overall system efficiency calculation, topologies that use smaller, more distributed approaches may have fewer losses (Fig. 11). The distributed architectures result in an increase in mass, due to the decrease in power density as power rating decreases. The effects of DEP are achieved by the number of propulsors, and the incurred mass may be minimized by using architectures with the largest power rating and fewest number of power system components that posses more propulsors.

In many cases, it may be desirable to include multiple redundant paths for power flow, so that the overall system reliability may be increased. In power systems, this is done through the addition of multiple redundant conductors between feeders and controlling the connections through a combination of breakers and relays. Each of these add mass to the EAPS, and therefore must be accounted for. A constant mass penalty for interconnection edges is included in the model to better represent effect of conductors and interfacing hardware such as circuit breakers. To further improve accuracy of this model, a designer may include geometry of an aircraft, voltage and current ratings to obtain exact conductor and interconnect specifications.

Two forms of energy storage are considered in this study, electrical and chemical. When jet fuel is burned, this exhibits a mass variability throughout a flight profile. In the case of a hybrid aircraft utilizing jet fuel or fuel cell technology, total aircraft mass decreases as it progresses throughout its flight. This variability may be significant in certain cases and is therefore included in this study. As the mass decreases, power requirements for various flight conditions will decrease as well. This is reflected in the total energy used calculations and output performance characteristics of the EAPS architecture.

\section{B. Design Candidate Survivability}

Power system architectures may also be evaluated based upon their survivability, meaning how well the system can

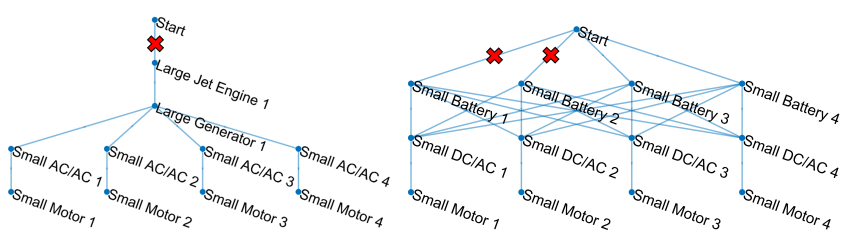

Figure 12. Illustration of topology survivability for designs considered in the optimization. Red markers on the graphs indicate minimum number of failures prior to flight power decreasing below 75 percent. Figure includes two designs selected from the pareto front when considering mass versus losses.

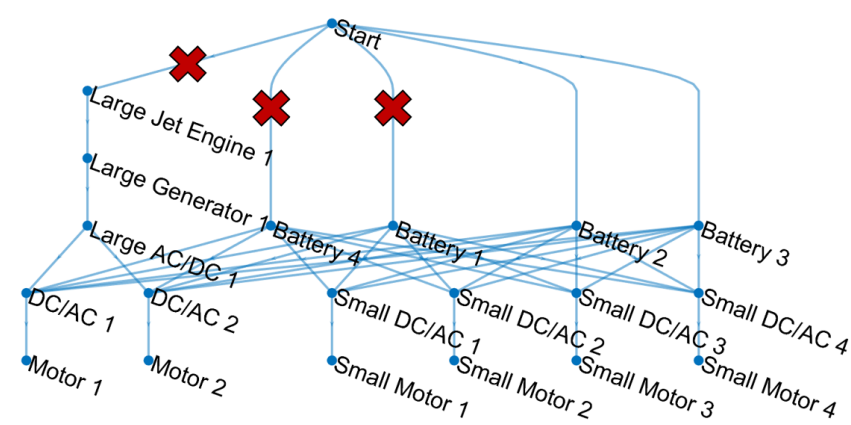

Figure 13. Electric aircraft power system topology that demonstrates N-3 survivability. While assurance of flight safety is increased by the addition of multiple redundant connections and components, overall system mass increases accordingly.

perform if components go offline. In this paper, survivability was scored using a tiered method, based on how many components may fail before thrust production capability falls below 75 percent of the nominal rated power of the case study.

A set of pareto front designs from the mass versus efficiency example discussed in the previous section was extracted and evaluated for their survivability (Fig. 12). Topologies which have a higher survivability index often exhibit greater mass, due to redundant components and connections. For example, a hybrid-electric architecture with $\mathrm{N}-3$ survivability may be achieved through the addition of many redundant feeders (Fig. 13).

Turbo-electric topologies with fewer system components may perform best when considering metrics such as overall power system mass and efficiency, as discussed in the previous section. However, topologies that incorporate either a distributed approach through the implementation of many smaller energy storage and propulsive units or many redundant connections tend to exhibit higher survivability.

The proposed framework may be expanded to analyze EAPS reliability using minimal cut sets to determine, for each node, the minimum series of events which need to occur before the node is inoperative. This information can then be converted into useful data such as the probability of failure. One challenge with the evaluation of power system reliability is obtaining accurate failure rates of components, which may be calculated using stress factors or specified by the manufacturer based on extensive laboratory testing. Additionally, estimating the interaction effects between components is non-trivial and must be carefully assessed, as seen in [50]. 


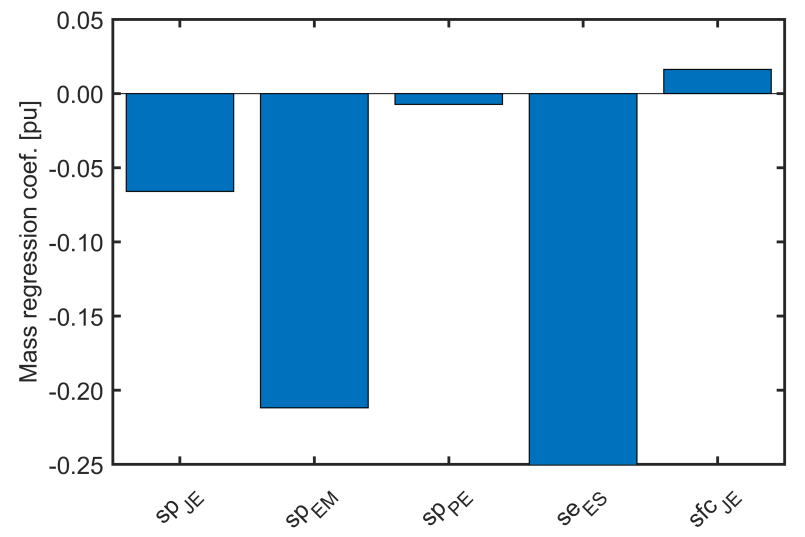

Figure 14. The regression coefficients in per unit associated with total power system mass resulting from numerical calculations for design candidate, D2. The regression coefficient for the specific energy for the electrical energy storage is -1.27 .

\section{Sensitivity Analysis}

As technology progresses, specific power, specific energy, and efficiency of the individual components used in the aircraft power system will advance accordingly. To evaluate the effect on performance metrics these advancements may have, a sensitivity study has been conducted for a selected topology design candidate, D2, which is a hybrid architecture with half of the required energy for the flight mission originating from electrical storage. This was done by fitting a regression curve using a second order polynomial:

$$
\begin{gathered}
Y=\beta_{0}+\sum_{i=1}^{d_{v}} \beta_{i} X_{C_{i}}+\sum_{i=1}^{d_{v}} \beta_{i i} X_{C i}^{2}+\sum_{i=1}^{d_{v}} \sum_{j=i+1}^{d_{v}} \beta_{i j} X_{C i} X_{C j}, \\
X_{C i}=\frac{x_{i}-\left(x_{i, \max }+x_{i, \min }\right) / 2}{\left(x_{i, \max }+x_{i, \min }\right) / 2} ; i=1,2, \ldots, d_{v}, \quad \text { (9) }
\end{gathered}
$$

where $Y$ is a response parameter; $\beta$, the regression coefficient; $d_{v}$, the number of factors, $x_{i}$, the $i^{t h}$ input factor; and $X_{C i}$, the normalized (coded) value of the $i^{\text {th }}$ factor. Factors may be normalized as shown in (9). $X_{C i}=0$ represents the specified values of the factors, and $\beta_{0}$ is a representation of response parameter in this reference situation. For each of the following sensitivity studies conducted, a single $500 \mathrm{~kW}$ parallel hybrid topology with 50 percent electrical energy storage as seen in design D3 was used. The factors and associated ranges of variation considered for the analysis were derived from that seen in the literature review conducted on the power system components shown in Table. I.

When considering mass as a primary objective for a given design candidate, there are five factors that affect the total power system mass: jet engine specific power, electric machine specific power, power electronics specific power, electrical energy storage specific energy, and jet engine specific fuel consumption. The results of this sensitivity study show that within the currently available technology, the greatest reduction of mass can be achieved by utilizing the highest performance energy storage with respect to specific power, as seen in Fig. 14.

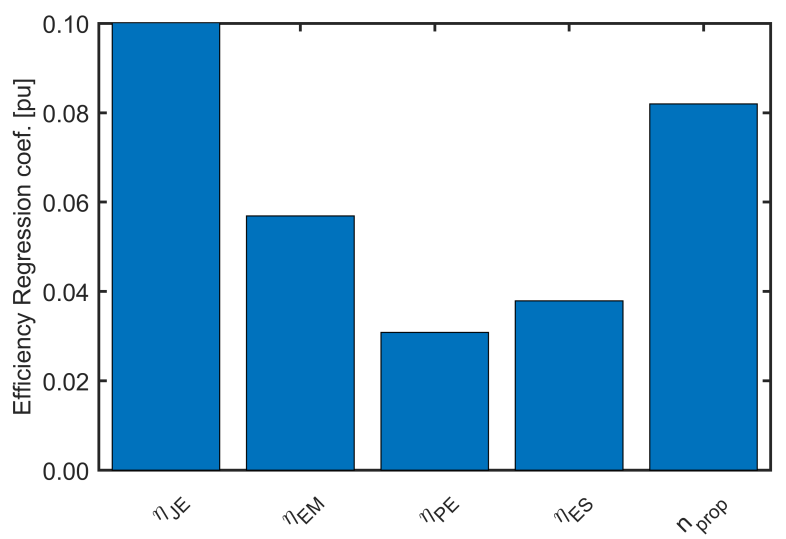

Figure 15. The regression coefficients in per unit associated with total power system efficiency resulting from numerical calculations for design candidate, D2. The regression coefficient for the efficiency of jet engines is 0.3 .

Table I

EAPS COMPONENT VARIABLES. VALUES STATED REFLECT THOSE SEEN

\begin{tabular}{|c|c|c|}
\hline $\begin{array}{l}\text { Component } \\
\text { Factors }\end{array}$ & & $\begin{array}{l}\text { Ref. values } \\
\text { (min, max) }\end{array}$ \\
\hline$s e_{E S}$ & $\begin{array}{l}\text { Energy storage specific } \\
\text { energy }[\mathrm{kWh} / \mathrm{kg}]\end{array}$ & $0.07,0.2$ \\
\hline$s p_{J E}$ & $\begin{array}{l}\text { Jet engine specific } \\
\text { power }[\mathrm{kW} / \mathrm{kg}]\end{array}$ & 1,11 \\
\hline$s f c_{J E}$ & $\begin{array}{l}\text { Jet engine specific } \\
\text { fuel consumption }[\mathrm{kg} / \mathrm{kWh}]\end{array}$ & $0.186,0.495$ \\
\hline$s p_{E M}$ & $\begin{array}{l}\text { Electric machine specific } \\
\text { power }[\mathrm{kW} / \mathrm{kg}]\end{array}$ & 1,16 \\
\hline$s p_{P E}$ & $\begin{array}{l}\text { Power electronic specific } \\
\text { power }[\mathrm{kW} / \mathrm{kg}]\end{array}$ & 11,34 \\
\hline$\eta_{J E}$ & Jet engine eff. [\%] & $16.23,43.20$ \\
\hline$\eta_{E M}$ & Electric machine eff. [\%] & 93,98 \\
\hline$\eta_{E M}$ & Power electronics eff. [\%] & 96,99 \\
\hline$\eta_{E M}$ & Energy storage eff. [\%] & 95,99 \\
\hline$n_{\text {prop }}$ & Number of propulsors & 2,8 \\
\hline
\end{tabular}
IN THE SURVEY CONDUCTED IN FIGS. 3-6.

Alternatively, when the primary objective is system efficiency, the five factors that influence the output are efficiencies of the jet engines, electric machines, power electronics, and energy storage and number of propulsors. This study shows that, given the current ranges of available component efficiencies, jet engines may have the greatest contribution to the total system losses (Fig. 15).

\section{CONCLUSION}

Electric aircraft designs today show large variations in power system types as well as number of components used. This paper presents developments toward an optimization approach for aircraft power systems which incorporate electric propulsion. The proposed approach is capable of evaluating thousands of design candidates based on performance metrics such as mass, efficiency, and survivability. 
This paper includes a study for an aircraft designed for a rated power of $500 \mathrm{~kW}$, intended for short-haul missions, such as those seen in commercial aviation. The case study power system candidate pool includes both conventional jet and electrical propulsion means, and steps of granularity in between degrees of electrical energy storage. Example outcomes include comparative evaluation of potential power system architectures with respect to mass, efficiency and survivability.

The results of this study show that aircraft with greater means of electrical energy storage exhibit higher efficiency, while those with more chemical energy storage and fewer components perform better when considering mass. Additionally, when the efficiency benefits of distributed propulsion was considered, designs with more, smaller propulsors may have a greater overall efficiency and prove to be more survivable, at the cost of an incurred mass. The framework developed provides a tool for system designers to evaluate tradeoffs between various EAPS topology candidates. While it may be apparent that purely electric designs are most efficient and turbo-electric architectures have the lowest mass, what is often needed is a trade-off, based on mission goals. It was demonstrated that the proposed automated optimization process is able to identify such best-case compromises represented by series and parallel hybrid architectures with various degrees of electrical energy storage and topology layouts, including multiple propulsors.

\section{ACKNOWLEDGMENT}

The support of this research by the National Aeronautics and Space Administration, through the NASA Grant no. KY GF-19-051, is gratefully acknowledged.

\section{REFERENCES}

[1] "E-fan," "April 30, 2014, accessed Sept. 10, 2020". [Online]. Available: https://newatlas.com/e-fan-airbus-electric-plane/31823/

[2] "Magnix," "June 8, 2020, accessed Sept. 10, 2020". [Online]. Available: https://www.thenakedscientists.com/articles/interviews/ all-electric-plane-takes-flight

[3] "E-fan X," "accessed Sept. 10, 2020". [Online]. Available: https://www. airbus.com/innovation/zero-emission/electric-flight/e-fan-x.html\#ove

[4] J. L. Kratz and G. L. Thomas, "Dynamic analysis of the starc-abl propulsion system," AIAA Propulsion and Energy 2019 Forum, 2019.

[5] M. J. Armstrong, C. A. H. Ross, M. J. Blackwelder, and K. Rajashekara, "Propulsion system component considerations for nasa $\mathrm{n} 3-\mathrm{x}$ turboelectric distributed propulsion system," SAE International Journal of Aerospace, vol. 5, no. 2, p. 344-353, 2012.

[6] M. A. Hannan, M. M. Hoque, A. Hussain, Y. Yusof, and P. J. Ker, "State-of-the-art and energy management system of lithium-ion batteries in electric vehicle applications: Issues and recommendations," IEEE Access, vol. 6, pp. 19362-19378, 2018.

[7] C. E. Jones, P. J. Norman, S. J. Galloway, M. J. Armstrong, and A. M. Bollman, "Comparison of candidate architectures for future distributed propulsion aircraft," IEEE Transactions on Applied Superconductivity, vol. 26, no. 6, pp. 1-9, Sep. 2016.

[8] M. J. Armstrong, M. Blackwelder, A. Bollmann, C. Ross, A. Campbell, C. Jones, and P. Norman, "Architecture, Voltage, and Components for a Turboelectric Distributed Propulsion Electric Grid," Rolls-Royce and Georgia Institute of Technology and University of Strathclyde, Tech. Rep., July 2015.

[9] J. C. Gladin, D. Trawick, C. Perullo, J. C. Tai, and D. N. Mavris, "Modeling and design of a partially electric distributed aircraft propulsion system with GT-HEAT," 55th AIAA Aerospace Sciences Meeting, 2017.

[10] H. Smith, D. Sziroczák, G. Abbe, and P. Okonkwo, "The GENUS aircraft conceptual design environment," Proceedings of the Institution of Mechanical Engineers, Part G: Journal of Aerospace Engineering, vol. 233, no. 8, p. 2932-2947, 2018.
[11] N. Chen, H. Wang, H. Li, and D. Xu, "Generic derivation of optimal architecture for a resilient microgrid with graph theory," in 2019 IEEE 10th International Symposium on Power Electronics for Distributed Generation Systems (PEDG), 2019, pp. 359-364.

[12] T. A. Trapp, "Shipboard integrated engineering plant survivable network optimization," Ph.D. dissertation, Massachusettes Institute of Technology, 2015.

[13] D. Lawhorn, V. Rallabandi, and D. M. Ionel, "A network graph technique for the design of electric aircraft power systems," in 2020 IEEE Transportation Electrification Conference Expo (ITEC), 2020, pp. 809813.

[14] S. Clarke, M. Redifer, K. Papathakis, A. Samuel, and T. Foster, "X57 power and command system design," in 2017 IEEE Transportation Electrification Conference and Expo (ITEC), June 2017, pp. 393-400.

[15] J. Felder, "Nasa n3-x with turboelectric distributed propulsion," NASA Glenn Research Center, Tech. Rep., November 2014. [Online]. Available: https://ntrs.nasa.gov/search.jsp?R=20150002081

[16] "Compact, quiet, low-vibration, high-efficiency rotary engines," 2018. [Online]. Available: https://liquidpiston.com/wp-content/uploads/2018/ 07/LiquidPiston-X-Specifications-2018-05-14-3.pdf

[17] "Zoche aero-diesels," 2000. [Online]. Available: http://www.zoche.de/ specs.html

[18] "Cd-200 jet-a enginer series," 2020. [Online] Available: http://www.continentalmotors.aero/uploadedFiles/Content/ Engines/Diesel_Engines/CD200-SpecSheet.pdfl

[19] "Helicopters." [Online]. Available: https://www.rolls-royce.com/ products-and-services/civil-aerospace/helicopters

[20] "Helicopter engines," 2019. [Online]. Available: https://www.pwc.ca/ en/products-and-services/products/helicopter-engines

[21] "Engines," 2020. [Online]. Available: https://aerospace.honeywell.com/ en/learn/products/engines

[22] "Gemini diesel engines." [Online]. Available: https://www.geminidiesel.aero/application/files/5014/2828/3029/ GEMINI_GenInfoSht_LowRez.pdf

[23] "Electric motor demonstrator sets two world records in two hours after five years of work," Nov 2019. [Online]. Available: https://car.osu.edu/news/2019/11/ electric-motor-demonstrator-sets-two-world-records-two-hours-after

[24] A. K. Yoon, D. Lohan, F. Arastu, J. Xiao, and K. Haran, "Direct drive electric motor for starc-abl tail-cone propulsor," AIAA Propulsion and Energy 2019 Forum, 2019.

[25] "Electric propulsion components with high power densities for aviation," 2015. [Online]. Available: https://nari.arc.nasa.gov/sites/ default/files/attachments/Korbinian-TVFW-Aug2015.pdf

[26] R. W. Dyson, R. H. Jansen, K. P. Duffy, and P. J. Passe, "High efficiency megawatt machine rotating cryocooler conceptual design," in 2019 AIAA/IEEE Electric Aircraft Technologies Symposium (EATS), 2019, pp. 1-15.

[27] "Yasa 750r," 2018. [Online]. Available: https://www.yasa.com/yasa-750/

[28] "Yasa p400r," 2018. [Online]. Available: https://www.yasa.com/ yasa-400/

[29] "1 megawatt power generator," 2019. [Online]. Available: https://aerospace.honeywell.com/content/dam/aero/en-us/documents/ learn/products/electric-power/brochures/N61-2229-000-000_ 1MW-Generator-br.pdf

[30] X. Zhang, C. L. Bowman, T. C. O'Connell, and K. S. Haran, "Large electric machines for aircraft electric propulsion," IET Electric Power Applications, vol. 12, no. 6, pp. 767-779, 2018.

[31] "E-motor," 2019. [Online]. Available: https://www.mclaren.com/applied/ products/item/e-motor-120kw-130nm/

[32] A. Yoon, Xuan Yi, J. Martin, Yuanshan Chen, and K. Haran, "A highspeed, high-frequency, air-core pm machine for aircraft application," in 2016 IEEE Power and Energy Conference at Illinois (PECI), 2016, pp. $1-4$.

[33] H. Lin, H. Guo, and H. Qian, "Design of high-performance permanent magnet synchronous motor for electric aircraft propulsion," in 201821 st International Conference on Electrical Machines and Systems (ICEMS), 2018, pp. 174-179.

[34] "Hvh410-150 electric motor," 2016. [Online]. Available: https: //cdn.borgwarner.com/docs/default-source/default-document-library/ remy-pds---hvh410-150-sheet-euro-pr-3-16.pdf?sfvrsn=a642cd3c 11

[35] Z. Zhang, W. Geng, Y. Liu, and C. Wang, "Feasibility of a new ironless-stator axial flux permanent magnet machine for aircraft electric propulsion application," CES Transactions on Electrical Machines and Systems, vol. 3, no. 1, pp. 30-38, 2019.

[36] "Emrax 348," 2020. [Online]. Available: https://emrax.com/e-motors/ emrax-348/ 
[37] E. R. Laithwaite, "The goodness of a machine," Electronics and Power, vol. 11, no. 3, pp. 101-103, 1965.

[38] “"magnidrive"," 2020. [Online]. Available: https://www.magnix.aero/ products

[39] "High performance electric motors and drives," "accessed Sept. 10, 2020". [Online]. Available: https://www.mclaren.com/applied/ case-study/relentless-drive-power-density-and-efficiency/

[40] "Cascadia motion - inverters," "accessed Sept. 10, 2020". [Online]. Available: https://www.cascadiamotion.com/inverters

[41] High Voltage Low Power Inverter, Borg Warner, 2019, hVLP-10, HVLP20

[42] "Yasa controllers," "accessed Sept. 10, 2020". [Online]. Available: https://www.yasa.com/controllers/

[43] D. Zhang, J. He, D. Pan, M. Dame, and M. Schutten, "Development of megawatt-scale medium-voltage high efficiency high power density power converters for aircraft hybrid-electric propulsion systems," in 2018 AIAA/IEEE Electric Aircraft Technologies Symposium (EATS), 2018, pp. $1-5$.

[44] M. Garrett, D. Avanesian, M. Granger, S. Kowalewski, J. Maroli, W. Miller, R. Jansen, and P. Kascak, "Development of an $11 \mathrm{kw}$ lightweight, high efficiency motor controller for nasa x-57 distributed electric propulsion using sic mosfet switches," in 2019 AIAA/IEEE Electric Aircraft Technologies Symposium (EATS), 2019, pp. 1-8.

[45] T. M. Indra Mahila, A. Jannifar, and M. Heikal Hasan, "Review of available methods and development on energy storage; technology update," Renewable and Sustainable Energy Reviews, vol. 33, no. 1, pp. 532-545, 2014.

[46] M. Farhadi and O. Mohammed, "Energy storage technologies for high-power applications," IEEE Transactions on Industry Applications, vol. 52, no. 3, pp. 1953-1961, 2016.

[47] T. Kadyk, C. Winnefeld, R. Hanke-Rauschenbach, and U. Krewer, "Analysis and design of fuel cell systems for aviation," Energies, vol. 11, no. 2, p. 375, Feb 2018. [Online]. Available: http: //dx.doi.org/10.3390/en11020375

[48] R. Rodrigues, Y. Du, A. Antoniazzi, and P. Cairoli, "A review of solidstate circuit breakers," IEEE Transactions on Power Electronics, vol. 36, no. 1, pp. 364-377, 2021

[49] D. Lawhorn, V. Rallabandi, and D. M. Ionel, "Scalable graph theory approach for electric aircraft power system optimization," in 2019 AIAA/IEEE Electric Aircraft Technologies Symposium (EATS), August 2019, pp. 1-5.

[50] Y. Zhao, Y. Che, T. Lin, C. Wang, J. Liu, J. Xu, and J. Zhou, "Minimal cut sets-based reliability evaluation of the more electric aircraft power system," Mathematical Problems in Engineering, vol. 2018, 2018.

[51] H.-J. Steiner, P. Vratny, C. Gologan, K. Wieczorek, A. Isikveren, and M. Hornung, "Optimum number of engines for transport aircraft employing electrically powered distributed propulsion," CEAS Aeronautical Journal, vol. 5, pp. 157-170, 062014

[52] D. Lawhorn, V. Rallabandi, and D. M. Ionel, "Electric aircraft system co-simulation including body, propeller, propulsion, and energy storage models," in 2019 IEEE Transportation Electrification Conference and Expo (ITEC), 2019, pp. 1-5.

[53] H. D. Kim, A. Perry, and P. J. Ansell, "A review of distributed electric propulsion concepts for air vehicle technology," in 2018 AIAA/IEEE Electric Aircraft Technology Symposium (EATS), 2018, pp. 1-21.

[54] A. Lundbladh and T. Grönstedt, "Distributed propulsion and turbofan scale effects," 2005. 